\title{
Challenges and solutions for scaling up social media-based HIV self-testing: Author's reply
}

Yi Zhou ${ }^{1, \S}$, Dan $\mathrm{Wu}^{2, \S}$, Hongbo Jiang ${ }^{3}$, Weiming Tang ${ }^{4,5,6 *}$

1. Zhuhai Center for Diseases Control and Prevention, Zhuhai, China

2. Department of Clinical Research, London School of Hygiene \& Tropical Medicine, London, the United Kingdom

3. Department of Epidemiology and Biostatistics, School of Public Health, Guangdong Pharmaceutical University, Guangzhou,

4. China Dermatology Hospital, Southern Medical University, Guangzhou, China

5. Institute of Global Health and STI Research, Southern Medical University, Guangzhou, China

6. University of Minnesota-Twin Cities, Minneapolis, United States

$\S$ These authors contributed equally to this manuscript.

Correspondence to:

Dr. Weiming Tang, Dermatology Hospital, Southern Medical University, and the University of North Carolina at Chapel Hill Project-China, 2 Lujing Road, Guangzhou, 510095, China; Email:

Weiming_tang@med.unc.edu. Tel: 8615920567132 


\section{Dear Editor:}

We thank Dr. Zhao and colleagues for their comments on our study ${ }^{1}$. In this letter, we would like to share our reflections on the social media-based HIVST among men who have sex with men (MSM) and responses to Zhao et al. We will also discuss what we learned from this implementation program, challenges, and mitigating strategies for future scale-up of social media-based HIVST.

We agree with Zhao et al. that social media can potentially enhance the uptake of HIVST which is gaining popularity in terms of HIV testing because of its convenience, privacy protection, and alleviation of fear of stigma. A combination of social media and HIVST holds promise to scale up HIV test coverage and to reach hidden key groups, especially in China. However, China also faces challenges in scaling up HIVST via social media.

First, the country is still in the early phase of introducing HIVST, and high-level policies and guidelines for expanding HIVST are still not widely adopted. China FDA approved one oral testing kit for home-based self-testing, nevertheless, HIV self-test kits with varying quality are commonly sold on e-commerce platforms, such as Taobao, a most popular online shopping website. Previous data suggested that HIVST kits commercially available on the market had a rather poor accuracy rate of $64.6 \%{ }^{2}$ It is important to ensure the supply of quality-ensured test kits for HIV self-testing programs, especially when the post-testing counseling is lacking for e-commerce driven self-testing. The health authorities should also strengthen their role in monitoring the market use of HIV self-test kits.

Second, the needs of the community-based organizations (CBO) in engaging in demand generation, self-testing kits distributing, and linkage to care. Lay health volunteers at the CBO play a central role in program implementation and scaling-up. They usually conduct HIV awareness campaigns, distribute the test kits, provide free counseling services and mental healthcare to newly identified cases, and improve linkage-to-care. However, LGBT CBOs mainly exist in economically better off or capital cities and community ownership are suboptimal in sexual health and HIV/STIs prevention programs in many underdeveloped regions, which limited the scale-up of HIVST in these areas. Despite that, the social mediabased approaches may help address the issues and improve the access to HIVST in some areas, without the engagement of local CBOs, the quality of services remains a concern. 
Multi-sectoral efforts for building collaborations between local governments, private partners, advocacy groups, and sexual minorities are needed to scale up social media-based HIVST via CBOs.

Third, social media-based HIVST alone may still have a limited effect in improving testing coverage, and innovative strategies are needed to reach the most needed populations. Wu et al's study adopted the secondary distribution approach that allowed an index participant to apply multiple HIVST test kits and encouraged distribution within their social networks, which reached a high proportion of naive HIV testers. In addition, improving the social networkbased distribution of HIVST by existing sexual health influencers may be another useful strategy. Our preliminary results suggested that, compared to non-influencers, being an influencer is more likely to request more HIVST kits (aRR 1.40, 95\% Cl 1.22-1.60), distribute more kits to people in their social network (aRR 2.07, 95\% Cl 1.59-2.69). Other innovative strategies, such as peer referral, monetary incentive, and pay-it-forward can also be used to improve social-media-based HIVST. ${ }^{3}$

Fourth, there is a need to improve linkage to care. Although our study had reached an extremely high testing result returning rate (1141 out of 1150 distributes kits were used and returned), linkage to care is still a challenge for the social media-based HIVST approach. We used a refundable deposit mechanism, which incentivized the index participants to return their test results as well as to convince alter testers to return the test results. This mechanism may have partly contributed to the high returning rate of self-testing results. It also allowed our CBO volunteers to verify the HIVST results by reading the uploaded photo which is often challenging in many other contexts. In a place where financial models are different, the incentive structure for participants to return the HIVST result needs to be appropriately adjusted to local contexts.

Lastly, strategies to improve testing validity are needed. Our implementation study indicated that 30 of the $1141(2.6 \%)$ returned test results were invalid, mainly due to user errors. Even our invalid rate was lower compared to a previous study in China (12/27, 44.4\% MSM had invalid results) ${ }^{4}$ and another in Kenya ( with an invalid rate of $\left.15.1 \%, 36 / 239\right)^{5}$, strategies that can be used to improve the correct use and reporting the results of the testing are needed. 


\section{Acknowledgments}

This work was supported by the National Key Research and Development Program of China [grant number 2017YFE0103800]; Academy of Medical Sciences and the Newton Fund [grant number NIF\R1\181020]; the National Nature Science Foundation of China [grant numbers 81903371, 81772240]; and Zhuhai Medical and Health Science and Technology Plan Project [grant number 20181117A010064].

\section{Conflicts of interests}

All authors have declared no conflicts of interests. 


\section{References}

1. Zhao R, Tao Y, Zhang L. Can social media-based self-testing be a new paradigm for HIV testing in China? Clinical Infectious Diseases 2020.

2. Tang W, Wu D. Opportunities and challenges for HIV self-testing in China. The lancet HIV 2018; 5(11): e611-e2.

3. LU Y, Ni Y, Li X, et al. Monetary incentives and peer referral in promoting digital network-based secondary distribution of HIV self-testing among men who have sex with men in China: study protocol for a three-arm randomized controlled trial. BMC Public Health 2020; 20(1): 911.

4. Wei C, Yan L, Li J, Su X, Lippman S, Yan H. Which user errors matter during HIV self-testing? A qualitative participant observation study of men who have sex with men (MSM) in China. BMC Public Health 2018; 18(1): 1108.

5. Kurth $\mathrm{AE}$, Cleland $\mathrm{CM}$, Chhun N, et al. Accuracy and Acceptability of Oral Fluid HIV Self-Testing in a General Adult Population in Kenya. AIDS and behavior 2016; 20(4): 870-9. 